\title{
Photoelectric properties of metal-porous silicon-silicon planar heterostructures
}

\author{
A.V. Brodovoi ${ }^{1}$, V.A. Brodovoi ${ }^{2}$, V.A. Skryshevskyi ${ }^{2}$, S.G. Bunchuk ${ }^{3}$, L.M. Khnorozok ${ }^{4}$ \\ ${ }^{\prime}$ Institute for Problems of Materials Science, Kyiv, Ukraine \\ ${ }^{2}$ T. Shevchenko Kyiv National University, Kyiv, Ukraine \\ ${ }^{3}$ Institute of Semiconductor Physics, 41 prospect Nauky, 03028 Kyiv, Ukraine \\ ${ }^{4}$ Nizhyn Pedagogical University, Nizhyn, Ukraine
}

\begin{abstract}
Dark and light I-V characteristics as well as spectral curves of planar Ni-porous silicon- $p$-Si-Ni heterostructures have been studied. It is shown that photogeneration in heterostructure occurs both in the region of thin porous silicon layer and $p$-Si base. Avalanche breakdown is observed in the heterostructure at applied voltage biases $V>8-10 \mathrm{~V}$ and $T=77 \mathrm{~K}$. The coefficient of multiplying at $V=11 \mathrm{~V}$ achieves 60 (dark) and 200 (light), which is close to that of silicon $n^{+}-p-i-p^{+}$avalanche photodiodes.
\end{abstract}

Keywords: porous silicon, heterostructure, Schottky barrier

Paper received 04.09.02; accepted for publication 17.12.02.

\section{Introduction}

The interest to the study of photoeffects in porous silicon (PS) appears first of all from prospects of optoelectronic applications. So, over the past decade, the various PS heterostructures have been elaborated and thoroughly studied. Among them, the light emitted diodes based on contact of semitransparent metal-PS [1-3] and ITO-PS [4], photodiodes based on Schottky contact [5,6], $p$ - $n$ junction silicon solar cells with PS antireflection and light scattering layers [7-9], MIS structures with inversion layers [10] should be noted. Besides, the photoelectrical phenomenon is often regarded as a useful tool to investigate mechanisms of current transport in heterostructures.

In contrast to study of light emission in PS, there is no so much publications devoted to the barrier photocurrent or photoconductivity of bulk PS in sandwich structures like metal-PS-Si $[3,6,11,12]$. The significant internal quantum effficiency of heterojunction photocurrent was mentioned [6]. The spectral curves of external quantum efficiency show that photogeneration can take place both in Si base and PS film [3,11,12]. However, the photoconductivity of planar structures has not yet studied widely.
In this work, we consider dark and photocurrents of planar PS structures on Si substrate and discuss parameters of avalanche breakdown at the applied voltage biases.

\section{Experimental}

The PS layers were formed by electrochemical etching of (100) $p$-Si wafers with resistivity of $0.4 \Omega \cdot \mathrm{cm}$, doped by boron up to $10^{17} \mathrm{~cm}^{-3}$. Before etching, Si surface was boiled in concentrated $\mathrm{HNO}_{3}$ solution and etched in $\mathrm{HF}$ solution to remove the native oxide. Electrochemical etching was performed in the solution of $\mathrm{HF}(48 \%): \mathrm{C}_{2} \mathrm{H}_{5} \mathrm{OH}: \mathrm{H}_{2} \mathrm{O}=2: 2: 1$ at the current of $J_{a}=$ $=10 \mathrm{~mA} / \mathrm{cm}^{2}$ up to form the $1.0 \mu \mathrm{m}$ PS layer. After etching, the patterns were rinsed in deionized water and oxidized in $\mathrm{NaNO}_{3}$ solution. For electrical measurements, two contacts of Ni film were deposited on PS layer. The distance between contacts varies within the range of $0.3-0.8 \mathrm{~cm}$. The measurements of dark and light $\mathrm{I}-\mathrm{V}$ curves as well as an external quantum efficiency were carried out at $77 \mathrm{~K}$, when the patterns were kept in liquid nitrogen. The dark resisitivity of samples is of the order of $(1 \ldots 3) 10^{7} \Omega$. 


\section{A.V. Brodovoi et al.: Photoelectric properties of metal-porous ...}

\section{Results and discussion}

The dark I-V curves of the elaborated planar structures exhibit: i) the full symmetry at the change of sign of applied voltage biases; ii) the slow current growth at small voltage biases $(U<4 \mathrm{~V})$ and iii) the exponential current growth at the voltage biases $U>4 \mathrm{~V}$ that is obeyed to the law $I_{d} \sim e^{-E_{0} / E}$, where $E_{0}=1.4 \cdot 10^{4} \mathrm{~V} / \mathrm{cm}$ (Fig. 1).

For planar Me-PS-p-Si-PS-Me structure (Fig. 2), the current is governed by the high resistive PS region at small applied voltage biases. At the subsequent increase of applied voltage, the current increases by exponential law due to the avalanche breakdown. Most of all, the avalanche breakdown occurs in the region within Ni contact and silicon base, here the PS thickness amounts to $10^{-4} \mathrm{~cm}$. Then the resistance drastically reduces between metal

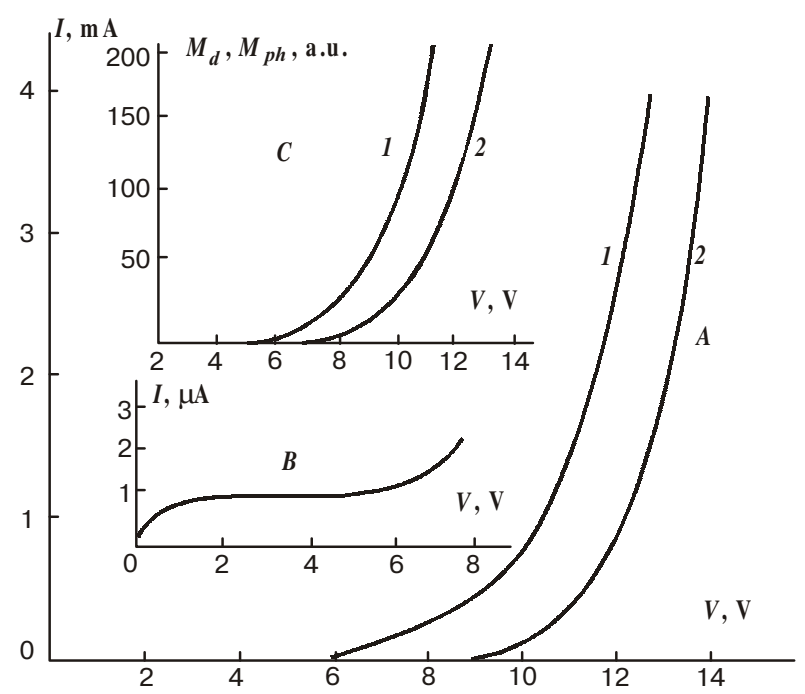

Fig. 1. $a-\mathrm{I}-\mathrm{V}$ curves of planar Ni-PS- $p$-Si-PS-Ni structure at $(I)$ and without illumination from tungsten lamp (2). $b$ - the dark I$\mathrm{V}$ curves in the region of small applied voltage biases. $c$ - the dependence of coefficients $M_{d}$ and $M_{p h}$ on applied voltage biases.

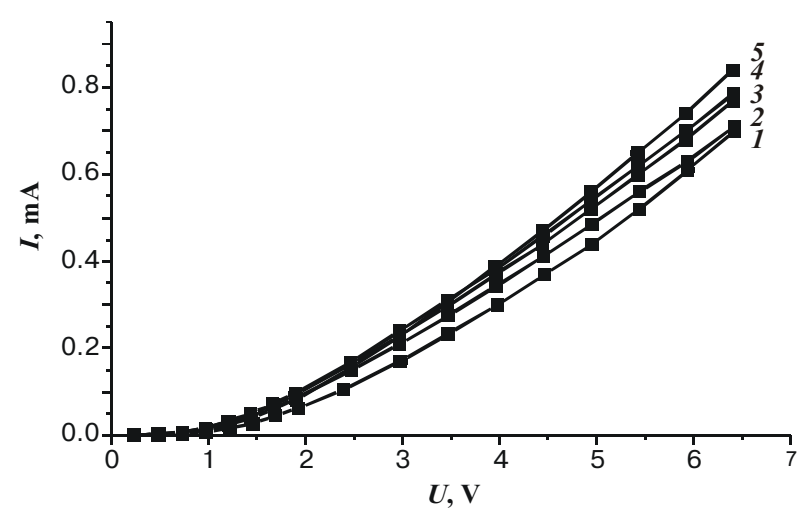

contact and $\mathrm{Si}$ base and the voltage drop redistributes between high resistive PS film and low resistive Si base. The evidence of shunt properties of Si base was obtained in the experiments with different gap between Ni contacts: the reduction of this distance does not practically change the sample resistivity.

The coefficient of multiplying at the given voltage $V$, $M(V)$ can be computed from I-V curves. For this purpose the segment of saturation of dark current (or photocurrent) should be extended towards the region of voltage at that the multiplying of electrons occurs (curve 2, Fig. 1). The coefficient of multiplying is calculated as the ratio of dark $I_{d}(V)$ (or photocurrent $I_{p h}(V)$ ) to the saturation current. Note, the shape of the obtained curves $M_{d}(V)$ and $M_{p h}(V)$ (curve 3, Fig. 1), is the same as for $n^{+}-p-i-p^{+}$avalanche photodiodes [13].

To justify the energetic diagram of proposed structure, we defined the parameters of Ni-PS-Si potential barriers. Fig. 3 shows the temperature change of forward and reverse $\mathrm{I}-\mathrm{V}$ characteristics of sandwich structure $\mathrm{Ni}$ $\mathrm{PS}(3 \mu \mathrm{m})-p-\mathrm{Si}-\mathrm{Ni}$. The height of potential barrier defined from the dependence of the saturation current on the temperature is amount to $0.38 \mathrm{eV}$, slowly depending on PS thickness. Then the energetic diagram of PS avalanche photodiodes includes two Schottky barriers switched towards each other. At the voltage of breakdown the ava-

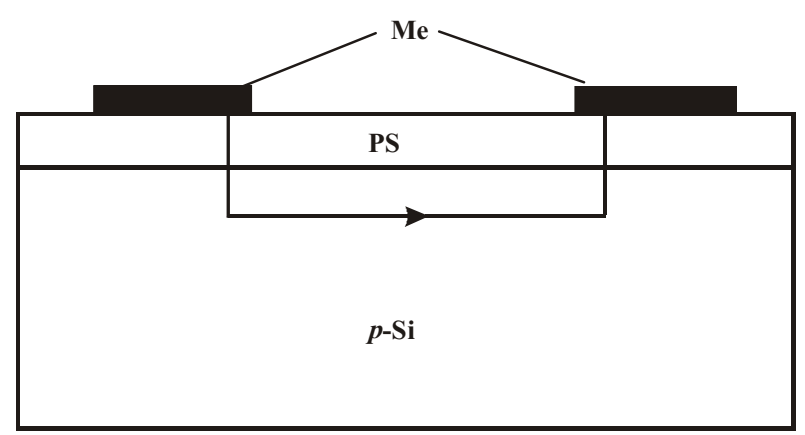

Fig. 2. Design of Me-PS-Si-PS-Me structure. Arrow shows the direction of current flow.

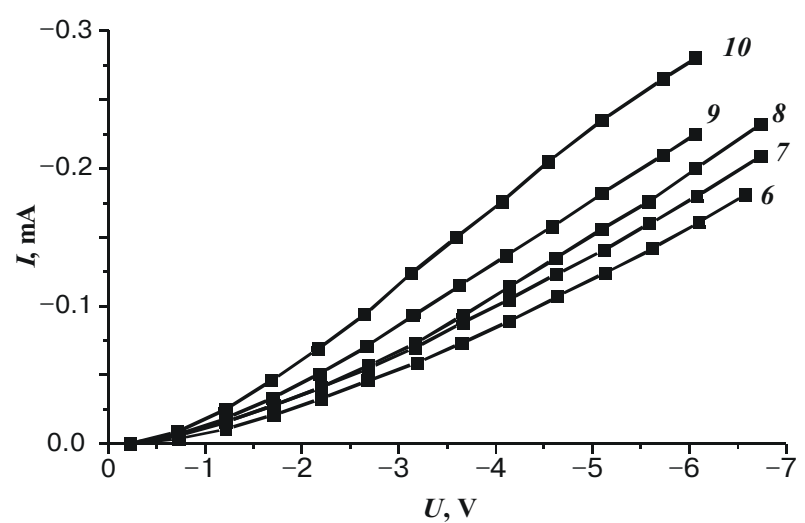

Fig. 3. Forward (1-5) and reverse (6-10) I-V curves for sandwich Ni-PS-p-Si-Ni structure at the following temperatures: $1-327$, $2-347,3-371,4-400,5-410,6-345,7-370,8-389,9-399,10-413 \mathrm{~K}$. 


\section{A.V. Brodovoi et al.: Photoelectric properties of metal-porous ...}

lanche starts in the depletion region near one of Ni contact switched in deep depletion mode. The subsequent increasing of applied voltage results in the widening of the region of electron multiplication on p-Si base (Fig. 4).

The spectral curve of photoconductivity is shown in Fig. 5. The spectrum shape does not depend on applied voltage. This spectrum shows the important contribution into the photogenaration both from the PS film (the maxi-

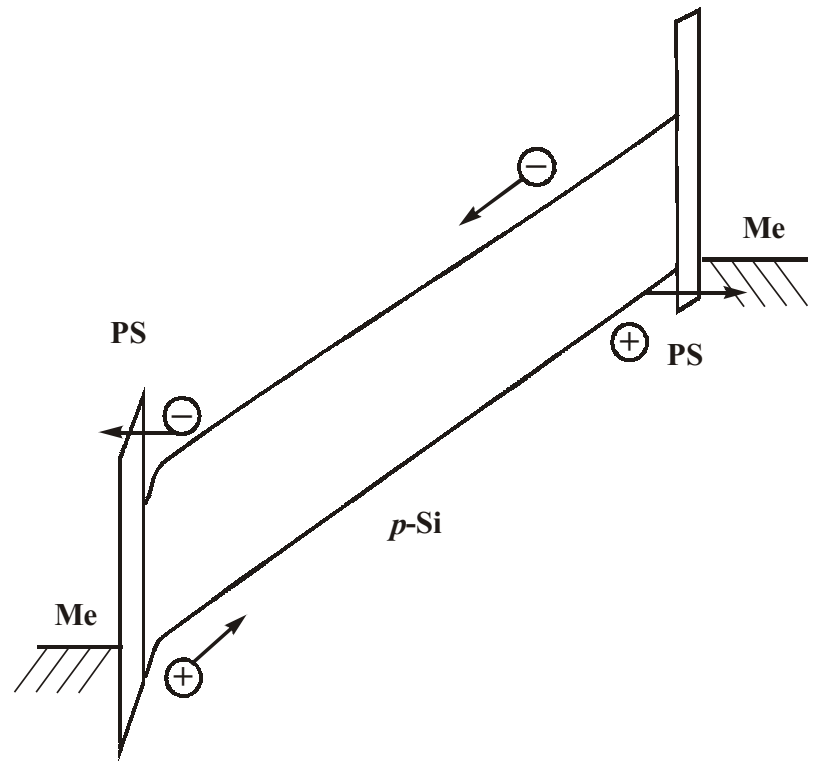

Fig. 4. The energetic scheme of Ni-PS- $p$-Si-PS-Ni heterostructure at the avalanche breakdown.

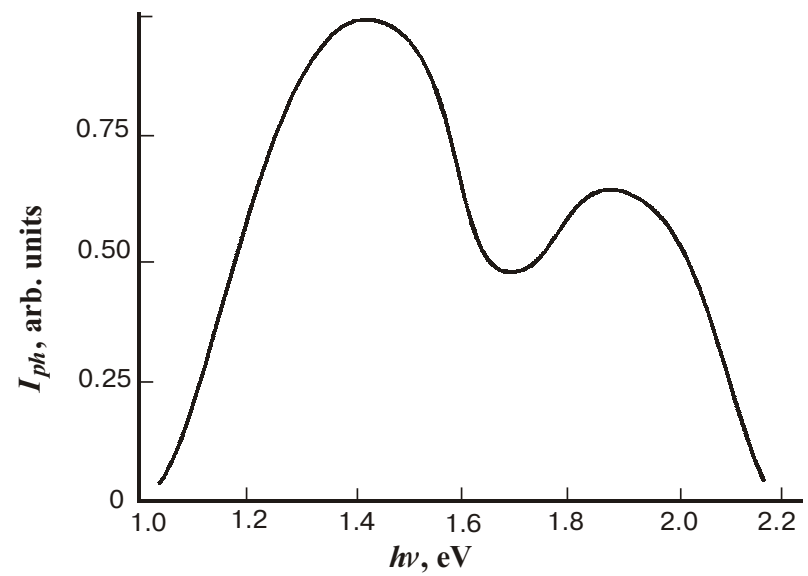

Fig. 5. The spectrum of photocurrent of Ni-PS-p-Si-PS-Ni structure. mum lies near $2.0 \mathrm{eV}$, which corresponds to the energy gap in PS) and $p$-Si (the long wavelength maximum lies near $1.5 \mathrm{eV})$. For the latter maximum, the photocurrent can be written as $I_{p h} \sim G(h v) \cdot\left[1-\exp (-\alpha W) /\left(1+\alpha L_{n}\right)\right]$, where $G(h v)$ is the photon flux, $\alpha=\alpha(h v)$ is the coefficient of optical adsorption in $\mathrm{Si}, \mathrm{W}$ is the width of depletion region in $\mathrm{Si}, L_{n}$ is the diffusion length of electrons [13]. This expresion satisfactory fits the long wavelength maximum of photocurrent spectrum. The photocurrent decreases at $h v>1.4 \mathrm{eV}$, which is stipulated by the recombination in the PS-Si interface. The surface recombination rate in PS-Si interface achieves $10^{5} \mathrm{~cm} / \mathrm{s}$ [9], which is less than for clear Si surface [14].

Thus, the planar Me-PS- $p$-Si-Me structures manifest the I-V characteristics close to those of typical avalanche photodiodes based $n^{+}-p-\mathrm{i}-p^{+}$structures. However, in order to compare the output parameters of PS avalanche photodiodes with another Si analogues, it is necessary to make supplementary measurements of the noise characteristics of PS heterostructures.

\section{References}

1. L.T. Canham, In: Frontiers of Nano-Optoelectronic Systems/ NATO Science Series. Eds L.Pavesi, E.Buzaneva., 6 (2000) 85.

2. A.Richter, W.Lang, S.Steiner, F.Kozlowski, H.Sandmaier. Mat. Res. Soc. Symp. Proc. 256 (1992) 209.

3. H.Shi, Y.Zheng, Y.Wang, R.Yuan, Appl.Phys.Lett. 63 (1993) 770 .

4. F.Namavar, H.P.Maruska, N.M.Kalkhoran. Appl.Phys.Lett. 60 (1992) 2514.

5. J.P.Zheng, K.L.Jiao, W.P.Shen, W.A.Anderson, H.S.Kwok. Appl.Phys.Lett., 61 (1992) 459.

6. C.Tsai, K.-H.Li, J.C.Campbell, A.Tasch, Appl. Phys. Lett., 62 (1993) 2818.

7. Y.S.Tsuo, Y.Xiao, M.J.Heben, X.Wu, F.J.Pem, S.K.Deb, Proc.23rd IEEE PVSC, Louisville, 1993, 287.

8. V.A.Skryshevsky, A.Laugier, Thin Solid Films, 346 (1999) 261.

9. V.A.Skryshevsky, A.Laugier, S.V.Litvinenko, V.I.Strikha, Proc. $2^{\text {nd }}$ World Conf. Exhibition on Photovoltaic Solar Energy Conversion, Wien, 1998, 1611.

10. V.A.Skryshevsky, Proc.2nd Int.Conf. Porous semiconductors-science and technology, Madrid, 2000, 178.

11. R.Sedlacik, F.Karel, J.Oswald, A.Fejfar, I.Pelant, J.Kocka, Thin Solid Films, 255 (1995) 269.

12. M.Ben-Chorin, F.Moller, F.Koch, J.Appl.Phys., 77 (1995) 4482 .

13. S.M.Sze, Physics of Semiconductor Devices, John Willey \& Sons, N.Y., 1981.

14. M.J.Keevers, F.W.Saris, G.C.Zhang, J.Zhao, M.A.Green, R.Elliman, Proc. 13th European Photovoltaic Solar Energy Conf, Nice, France, 1995. 